\title{
Technè
}

La science au service de l'histoire de l'art et de la préservation des biens culturels

$42 \mid 2015$

Science et conservation

\section{La restauration au Louvre du retable du Jugement dernier de Roger van der Weyden (1876-1878) : un rare cas de documentation photographique}

The restoration at the Louvre of Rogier van der Weyden's altarpiece The Last Judgement (1876-1878): a rare case of photographic documentation

\section{Claire Gerin-Pierre et Isabelle Cabillic}

\section{OpenEdition}

Journals

Édition électronique

URL : http://journals.openedition.org/techne/6647

DOI : 10.4000/techne.6647

ISSN : 2534-5168

Éditeur

C2RMF

Édition imprimée

Date de publication : 1 décembre 2015

Pagination : 46-54

ISBN : 978-2-7118-6249-8

ISSN : 1254-7867

\section{Référence électronique}

Claire Gerin-Pierre et Isabelle Cabillic, «La restauration au Louvre du retable du Jugement dernier de

Roger van der Weyden (1876-1878) : un rare cas de documentation photographique », Technè [En ligne], 42 | 2015, mis en ligne le 01 décembre 2015, consulté le 11 mars 2021. URL : http:// journals.openedition.org/techne/6647 ; DOI : https://doi.org/10.4000/techne.6647

\section{cc) (†)}

La revue Technè. La science au service de l'histoire de l'art et de la préservation des biens culturels est mise à disposition selon les termes de la Licence Creative Commons Attribution - Pas d'Utilisation Commerciale - Pas de Modification 4.0 International. 


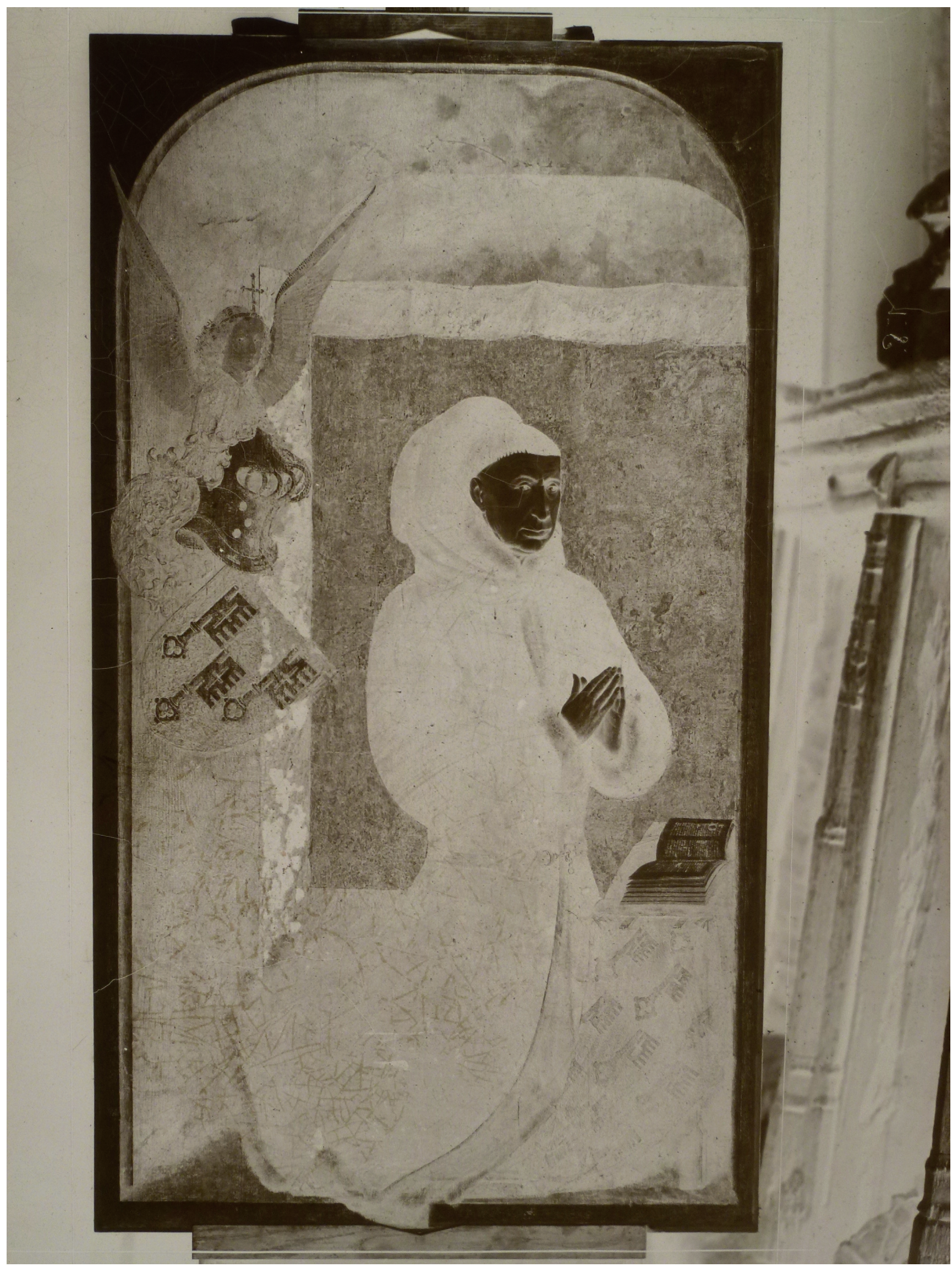

Fig. 1. Le Chancelier Rolin, après restauration, plaque de verre, 1882. @ Archives des Hospices civils de Beaune/Claire Gerin-Pierre. 
Claire Gerin-Pierre Avec la collaboration d'Isabelle Cabillic

\section{La restauration au Louvre du retable du Jugement dernier de Roger van der Weyden (1876-1878) : un rare cas de documentation photographique}

The restoration at the Louvre of Rogier van der Weyden's altarpiece The Last Judgement (1876-1878): a rare case of photographic documentation

Résumé. En France, les restaurations des tableaux des collections publiques ont donné lieu à la production d'un certain nombre de documents écrits dès le XVIII siècle. Ces documents, essentiellement comptables, sont encore nos principales sources pour reconstituer l'histoire matérielle des cuvres tout au long $d u$ XIXe siècle. Les rapports de restauration n'existent pas encore véritablement, et les témoignages visuels manquent la plupart du temps. Il faudra attendre la fin de la Première Guerre mondiale pour voir se dessiner une véritable évolution vers une documentation visuelle plus systématique des restaurations, avec le recours aux techniques nouvelles d'imagerie, puis les années 1950 pour voir la mise en place progressive de dossiers de restauration et d'une couverture photographique systématique. C'est pourquoi la restauration du retable de Roger van der Weyden, au Louvre à la fin du XIXe siècle, qui a donné lieu à une importante documentation écrite et visuelle, représente un cas rare et novateur.

Mots-clés. Documentation, archives, dossier, histoire de la restauration, constat, procès-verbal, photographie, radiographie, Villot, Reiset, Briotet, Chapuis, van der Weyden, Beaune.
Abstract. In France, since the 18th century, the restoration of paintings belonging to national collections has given rise to the production of a certain number of written documents. Mostly financial in nature, these documents remain our main source of information for piecing together the material history of works throughout the 19th century. Restoration reports did not really exist at this point, and visual records were lacking most of the time. Not until the end of World War I did systematic visual documentation of restoration campaigns begin to emerge, using new imaging techniques. Files on each restoration programme, including systematic photographic documentation, gradually became standard practice in the 1950s. That is why the restoration at the Louvre, in the late 19th century, of Rogier van der Weyden's altarpiece, which generated extensive written and photographic documentation, represents a rare and innovative case.

Keywords. Documentation, archives, file, history of conservation, report, photography, X-ray, Villot, Reiset, Briotet, Chapuis, van der Weyden, Beaune.

\section{Rappel des pratiques documentaires en matière de restauration des peintures aux XVIII ${ }^{\mathrm{e}}$ et XIX ${ }^{\mathrm{e}}$ siècles}

Les archives anciennes ont conservé peu de traces des interventions de restauration avant le XVIII ${ }^{\mathrm{e}}$ siècle. Les premiers inventaires, la plupart du temps, ne mentionnent pas les traitements opérés sur les œuvres, mis à part les changements de format. Mais avec l'émergence de la profession de restaurateur au cours du XVIII ${ }^{\mathrm{e}}$ siècle et l'ouverture progressive des collections au public, un certain nombre de documents commencent à témoigner des restaurations. Il s'agit avant tout des devis de restaurateurs et des mémoires correspondants, qui permettent à l'administration royale, puis révolutionnaire, de mieux contrôler le travail des restaurateurs. S'y ajoutent rapidement des constats d'état, qui justifient les opérations à entreprendre (et le prix exigé). Ce dernier type de document, très précieux pour témoigner de l'état des peintures à un instant donné, devient un élément indispensable à la bonne pratique de la restauration ${ }^{1}$. Enfin, le restaurateur doit décrire brièvement ses interventions dans son mémoire. On peut donc dire qu'on dispose désormais de détails sur les restaurations, même si on ne peut parler encore de rapports ${ }^{2}$.

Toutes ces mesures se révèlent d'autant plus nécessaires que les tableaux, devenus patrimoine national, peuvent faire désormais l'objet d'un débat public, et que l'administration peut se trouver mise en accusation à ce sujet. Au tout début de 1798, l'affaire Marin oblige l'administration à réfuter un certain nombre d'accusations ${ }^{3}$, et les constats réalisés se révèlent alors précieux. De même, avec l'afflux des saisies révolutionnaires exposées au musée du Louvre, on constate une certaine suspicion, particulièrement à l'étranger, sur les restaurations faites à Paris, voire des critiques assez sévères ${ }^{4}$. C'est dans ce contexte que l'on peut placer la production d'un document très complet, mais qui demeurera longtemps exceptionnel, le rapport très détaillé sur la restauration de la Madone de Foligno de Raphaël (avec constat des altérations, description minutieuse des interventions et explications des effets des 
produits employés par les chimistes membres de la commission assistant à l'opération de transposition de bois sur toile) ${ }^{5}$. Mais les autres très nombreuses restaurations pratiquées au Louvre n'ont pas donné lieu alors à de semblables publications, et on peut également remarquer que ce rapport ne comporte aucune illustration, aucun schéma qui permettrait de visualiser les lacunes, et donc de fait les ajouts à l'original.

Tout au long du XIX ${ }^{\mathrm{e}}$ siècle, la restauration des tableaux des musées continue d'être documentée essentiellement par des pièces comptables (devis, mémoires ou paiements à la journée), auxquelles on peut ajouter pour le Louvre les procès-verbaux de l'administration sous le Consulat ${ }^{6}$, des extraits de la correspondance des directeurs, les rapports des commissaires-experts sous la Restauration et la Monarchie de juillet $^{7}$, puis les comptes-rendus des commissions de restauration (en 1861, puis à partir de 1882) ${ }^{8}$. Tous ces documents ne comportent ni croquis ni dessins et sont souvent assez sommaires $^{9}$; de plus, comme l'usage des constats détaillés est souvent moins rigoureux que pendant la période révolutionnaire, il n'est pas toujours aisé de se faire une idée de l'état des œuvres avant restauration, et surtout de l'importance des interventions, particulièrement pour la couche picturale. On peut également observer que les traités de restauration, qui se multiplient au cours du XIX ${ }^{\mathrm{e}}$ siècle $^{10}$, ne mentionnent pas non plus à cette époque la nécessité de relever visuellement les altérations ou de documenter après restauration les zones repeintes. Il se peut que les restaurateurs aient pratiqué ce type de relevés, mais les archives publiques ne les conservent pas, et nous avons retrouvé actuellement trop peu d'archives privées de restaurateurs pour connaître réellement leur pratique en la matière.
Avec l'amélioration des techniques photographiques, on voit cependant émerger l'idée d'utiliser ponctuellement cet outil, qui présentait toutes les garanties d'exactitude et d'objectivité requises pour documenter l'état des œuvres, mais les mentions sont encore rares. Nous en avons retrouvé un exemple dans des notes écrites par Frédéric Villot en 1861, alors qu'il était encore conservateur des peintures au musée du Louvre. Il y rappelle la mutilation subie par un plafond de Véronèse, Jupiter foudroyant les Vices (inv. 147), placé autrefois à Versailles, où l'on avait cru bon de découper les figures et de les disposer de manière arbitraire sur un plus grand fond ${ }^{11}$. Il n'y a plus trace de ces mutilations depuis la restauration faite au Louvre, mais Villot regrette que «forts de notre loyauté, nous n'avons pris aucune précaution afin de faire constater qu'on avait osé découper avec des ciseaux les figures d'un chef-d'œuvre pour les disposer d'une manière arbitraire sur un fond agrandi ». Et comme le Louvre s'apprête alors à faire restaurer un autre plafond de Véronèse, là aussi modifié pour le château de Versailles, Saint Marc couronnant les Vertus théologales (inv. 148), il demande, « pour garder la mémoire d'un fait inqualifiable,

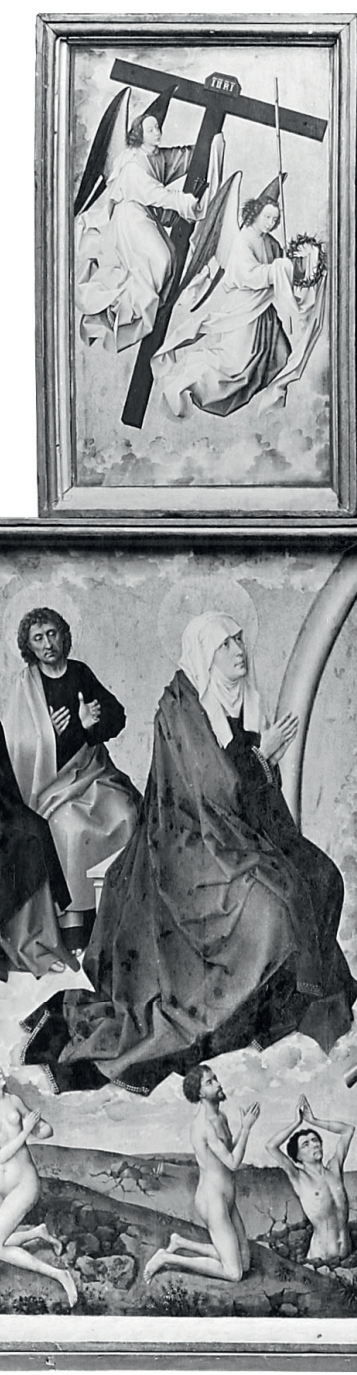


alors pour heureuse conséquence de susciter une abondante correspondance entre Frédéric Reiset et le président de la commission des Hospices, Louis Cyrod. Celle-ci est riche de précieux renseignements sur le déroulement de la restauration et les choix à faire en la matière. Décrivant les interventions à distance, Reiset se livre à des explications détaillées que nous n'avons pas ordinairement ${ }^{16}$. Et pour la même raison, les panneaux ont été photographiés avant et après intervention, cas très rare à l'époque.

Cette correspondance a de plus eu la chance d'être conservée entièrement, à la fois dans les archives du Louvre ${ }^{17}$, et dans un recueil spécialement consacré à la restauration du retable, conservé aux Archives des hospices civils de Beaune ${ }^{18}$ (fig. 3). L'on peut noter ici également la volonté, au Louvre et à Beaune, de bien conserver la mémoire de cette importante restauration. Le recueil de Beaune est particulièrement complet, car, outre la correspondance, il contient les copies des délibérations de la commission des Hospices, les lettres et mémoires des restaurateurs, les factures des photographes, le classement du retable au titre des monuments historiques, et quelques pages de souvenirs rédigées plusieurs années plus tard par un témoin de la restauration. De plus, tout a été soigneusement classé par ordre chronologique et réparti en cinq parties. On peut considérer ce recueil comme l'un des premiers dossiers de restauration complets dont on dispose, surtout si on y joint les photographies réalisées tout

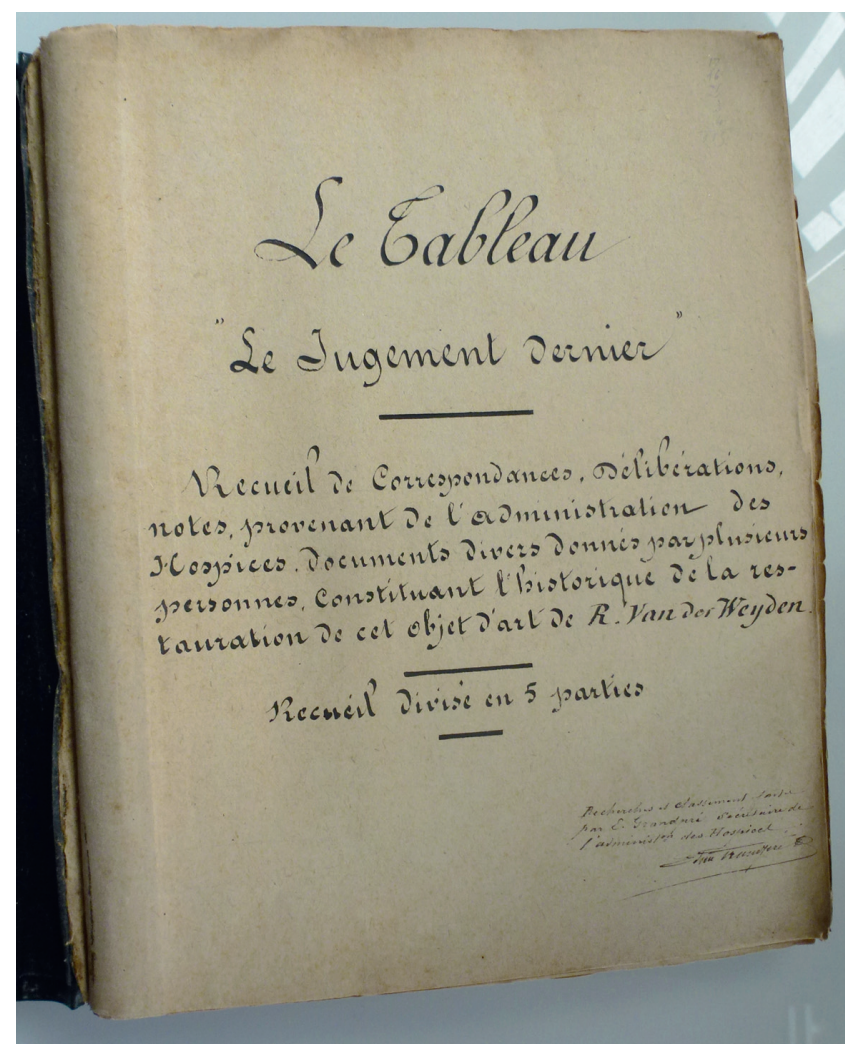

Fig. 3. Recueil documentant la restauration du retable de R. van der Weyden. (C) Archives des Hospices civils de Beaune/Claire GerinPierre. particulièrement à l'occasion de la restauration, conservées elles aussi pour la plupart. Il existe un jeu, en mauvais état, des panneaux avant intervention, conservé dans la documentation du Louvre, et un autre, en meilleur état, dans les archives des Hospices civils de Beaune ${ }^{19}$; il existe enfin un jeu de bien meilleure qualité (presque complet sauf deux panneaux) à la Médiathèque de l'architecture et du patrimoine ${ }^{20}$. Les archives des Hospices de Beaune conservent par ailleurs les plaques de verre et les tirages réalisés après la restauration du retable ${ }^{21}$ (fig. 1).

Dès l'envoi du premier panneau en 1875, la commission des Hospices décide en effet de le faire photographier à Beaune avant son départ ${ }^{22}$. Après sa restauration, Reiset informe Cyrod qu'il a fait photographier l'œuvre restaurée et qu'il va envoyer les épreuves ${ }^{23}$.

En 1877, lors de l'envoi des autres éléments du retable, Cyrod demande encore des photographies : «Vous déciderez comme la première fois les panneaux qu'il convient de reporter sur toile (...) Vous aurez à examiner aussi s'il est utile de faire photographier les panneaux avant leur réparation ${ }^{24}$. »Et Reiset, dans sa réponse, évalue le coût de la restauration et mentionne, dans les frais supplémentaires à envisager, les photographies. Puis il affirme : "La première chose que je ferai sera de faire photographier le tout dans l'état actuel, et plus tard probablement dans le cours du travail nous recommencerons ${ }^{25}$."

Le 3 avril 1877, il écrit donc à Cyrod : «J'ai, comme cela avait été convenu, commencé par faire faire les photographies des 13 panneaux. Vous recevrez par le chemin de fer ces 13 photographies, qui ont été bien exécutées (...) je vous envoie l'une des suites et je garde la seconde pour servir à notre travail (...) je crois d'ailleurs que vous ferez bien d'attendre pour faire faire un tirage sérieux que les travaux de restauration soient terminés, et j'ai lieu d'espérer qu'il y aura alors une amélioration sérieuse comparée à l'état actuel (...). » Enfin, en envoyant la facture du photographe, il s'excuse du coût mais insiste sur « cette reproduction photographique qu'il m'était impossible de vous éviter. Car avant tout il fallait constater l'état des panneaux ${ }^{26}$ "(fig. 4). Dans cette lettre, particulièrement intéressante pour notre propos, on voit bien que Reiset considère la documentation de l'œuvre avant restauration comme fondamentale, et qu'il va l'utiliser pour la suite des opérations (il garde une série de clichés « pour servir à notre travail »).

Enfin, d'autres photographies très intéressantes sont mentionnées dans cette correspondance, qui n'ont finalement pas été réalisées, mais qui montrent une intuition de l'utilité de la photographie pour documenter des éléments apparus fortuitement et dont on n'aura plus de témoignages ensuite : ici, les esquisses visibles sur la préparation, découvertes au moment de la transposition des panneaux. Cyrod écrit en effet : «Il nous a été en outre expliqué que par suite du grattage de la couche de craie qui sert de base à la peinture, on avait pu retrouver en certains endroits l'esquisse originale du peintre, avec ses modifications et ses tâtonnements ; ne seraitce pas là le cas, Monsieur, de tenter la reproduction par la 


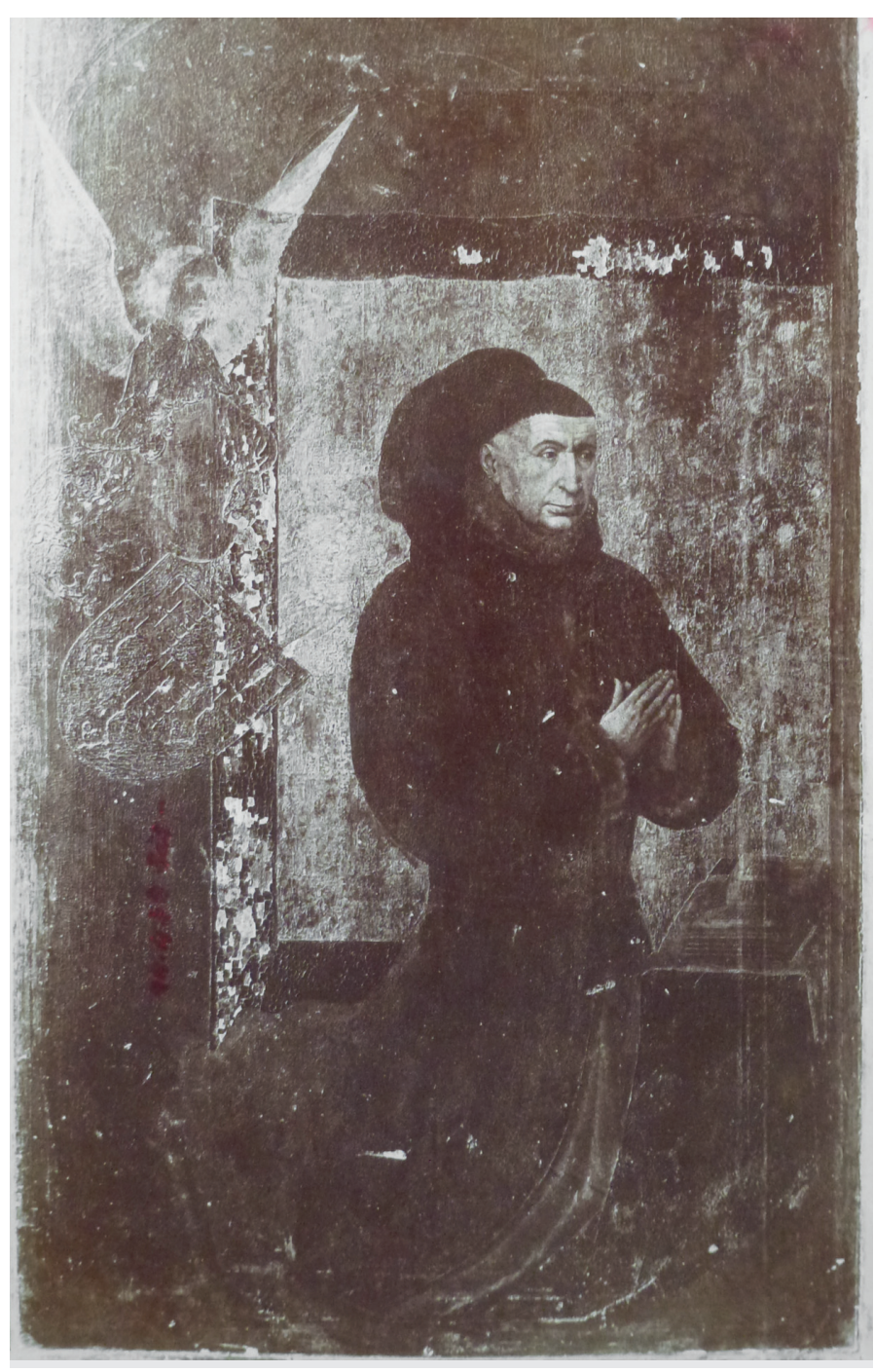

Fig. 4. Le Chancelier Rolin avant restauration, 1877 . (c) Claire Gerin-Pierre.

qu'il n'a rien vu d'intéressant à photographier dans ces « ébauches » (l'image était peut-être trop indistincte), et les photographies n'ont pas été faites. Sa mauvaise santé (à laquelle il est fait allusion dans ses dernières lettres) a peut-être aussi été responsable de cette décision, comme le suggère M. Bourgeois.

Nous disposons donc exceptionnellement pour cette œuvre de témoignages visuels sur son état, qui parfois justifient certaines interventions (l'enlèvement des repeints de pudeur sur les corps nus, repeints dénués de toute valeur artistique et couvrant largement des surfaces en bon état, comme l'expliquait Reiset dans sa correspondance), ou du moins expliquent certains choix (la vision des panneaux très dégradés et soulevés explique la décision de les transposer de bois sur toile, opération que l'on ne ferait plus aujourd'hui mais qui paraissait alors le meilleur remède). Les protagonistes de cette restauration sont bien conscients de l'utilité de cette documentation. Reiset rappelle que, sans ces pho-

photographie de ces esquisses ou fragments d'esquisses ? Il semble qu'on pourrait retrouver par la comparaison de ces ébauches avec le tableau lui-même quelques-uns des secrets du maître; en tous les cas, elles serviraient de documents pour l'histoire de la peinture. » Et un document inédit trouvé dans le recueil des archives des Hospices civils de Beaune éclaire cette demande. Un certain M. Bourgeois a rédigé en effet en 1892 quelques souvenirs sur cette restauration : «Pendant que M. Chapuis enlevait avec un rabot tout le bois d'un panneau qui devait être reporté sur toile, des dessins à l'encre de chine, première esquisse du peintre, apparurent sous la peinture. Ces dessins étaient complets, avec les repentirs ${ }^{27}$. M. Briotet eut la pensée de les faire photographier séance tenante. Quelle belle occasion d'avoir des dessins autographes de Roger van der Weyden ! Il en parla à M. Reiset, mais celuici ne voulut rien faire sans un avis spécial de l'administration. » Après un échange de correspondance, Reiset, malade et pressé de voir se terminer la restauration, ne donna pas suite et « les dessins un instant exhumés furent ensevelis de nouveau entre la toile et la peinture ${ }^{28}$ ». Reiset répond de fait dans sa lettre tographies, on aurait peine à croire que l'on ait eu un jour l'idée de défigurer ainsi le chef-d'œuvre du maître flamand et justifie par la même occasion la disparition de ces repeints malencontreux : «Si je n'avais vu la chose de mes yeux et si je n'avais pas fait faire, avant de commencer, la photographie du tableau, je n'aurais pu croire à une pareille audace ${ }^{29}$. » (fig. 5). Et le restaurateur Briotet, au moment de se faire payer, demande une somme légèrement plus élevée que prévu en évoquant l'importance des dégradations et le contraste entre l'état du retable avant son intervention et le résultat qu'il a obtenu : «En effet, j'ai eu plus de restaurations à faire que je le prévoyais, surtout pour le panneau Le paradis, comme la photographie peut le justifier ${ }^{30}$. » (fig. 6).

Outre la conservation et l'archivage de nombreux documents écrits, on constate donc ici un usage assez important de la photographie pour documenter la restauration du retable, et pour valider les interventions réalisées. Mais faute d'autres exemples, on ne peut affirmer que cette pratique ait alors été très répandue, en partie sans doute en raison du coût alors important des photographies ${ }^{31}$. 


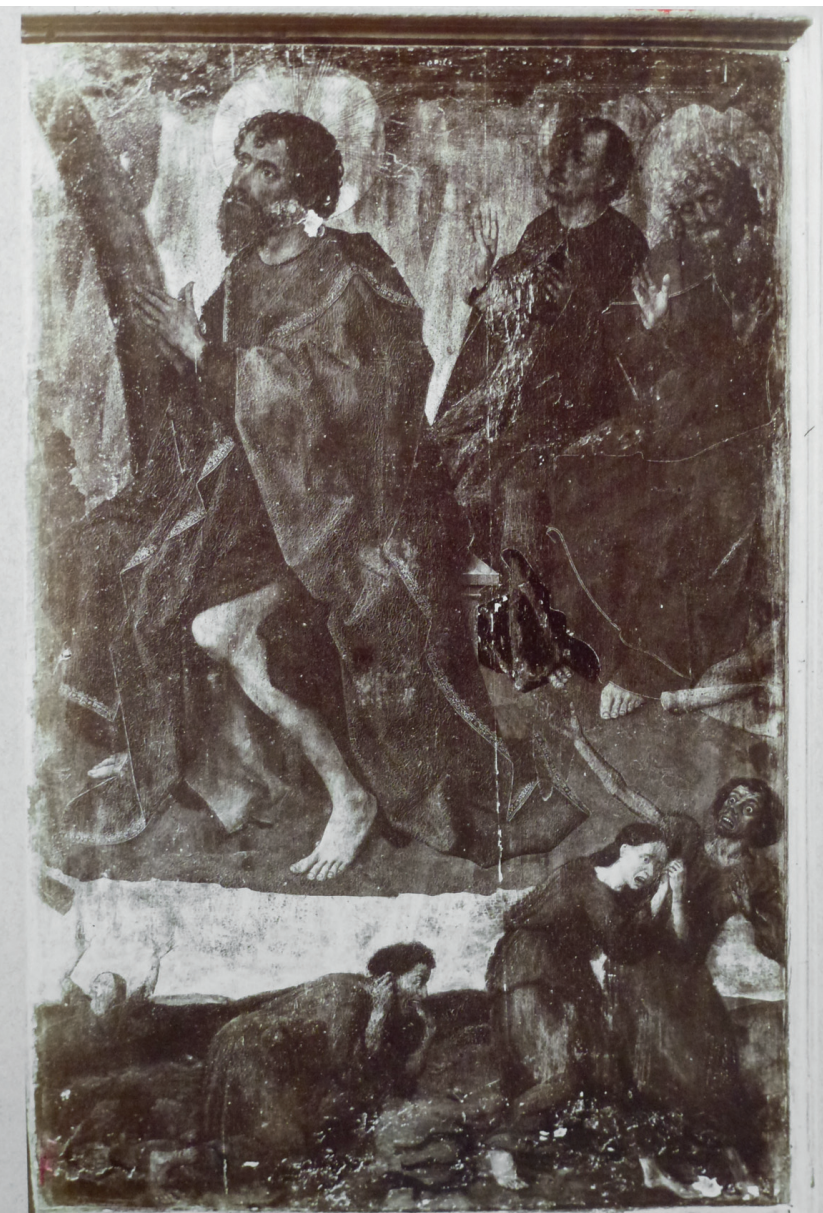

Fig. 5. Saint Jean-Baptiste, avant restauration, 1877. (c) Claire Gerin-Pierre.

\section{Vers un usage plus important de la documentation matérielle des œuvres}

Il faut attendre en fait la Première Guerre mondiale, et surtout les années qui suivent, pour voir se développer le recours à la photographie et aux nouvelles techniques d'imagerie. Le restaurateur Jean-Gabriel Goulinat, devenu rapidement expert des musées (avant de diriger l'atelier du Louvre à partir de 1935) s'exprime à ce sujet dès la Première Guerre mondiale, lors de son affectation au centre de radiologie de Tours en 1915 : « Le docteur Ledoux-Lebard, qui dirigeait ce laboratoire, m'avait pris en amitié. "Si vous voulez utiliser ces appareils pour des expériences sur la peinture, me dit-il, n’hésitez pas !" J'ai commencé dès lors et je crois avoir été le premier à examiner les tableaux aux rayons $\mathrm{X}$ et aux rayons ultraviolets, avant de restaurer une toile ${ }^{32}$.»

L'Institut Mainini, créé au Louvre dans l'entre-deuxguerres suite à une initiative personnelle, promeut lui aussi

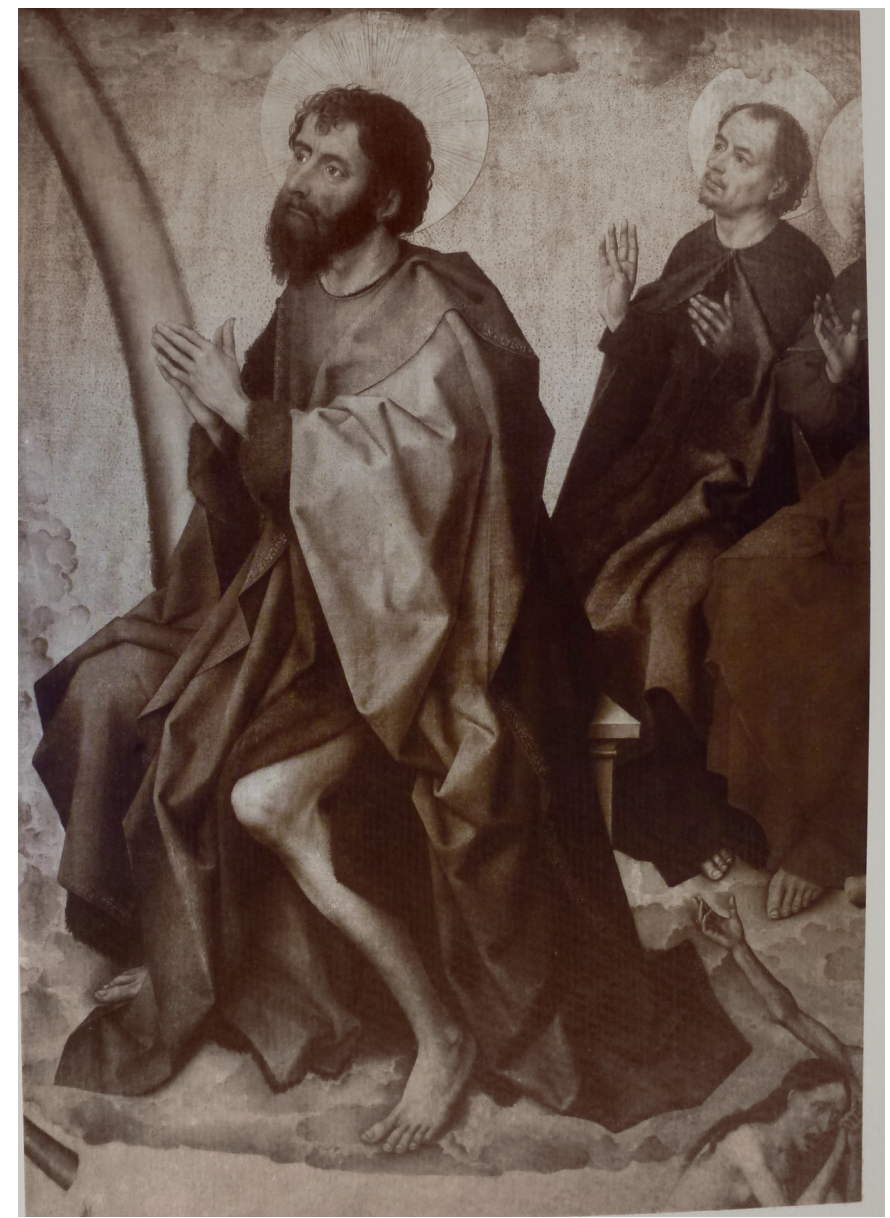

Fig. 6. Saint Jean-Baptiste, après restauration, 1882.

(c) Archives des Hospices civils de Beaune/Claire Gerin-Pierre.

l'utilisation régulière des rayons $\mathrm{X}$ et des ultra-violets pour une meilleure connaissance des œuvres et de leurs altérations. Enfin Goulinat, s'exprimant à Rome en 1930 lors de la Conférence internationale pour l'étude des méthodes scientifiques appliquées à l'examen et à la conservation des peintures, insiste sur l'apport de la radiologie, de la photographie (en particulier des ultra-violets) et de la macrophotographie dans la connaissance des œuvres et l'appréhension de la restauration. C'est lors de ces années que se constitue aussi un véritable suivi des œuvres sous forme de dossiers (au moins pour les plus importantes) rapportant les avis de la commission de restauration, les photographies avant et après restauration, les radiographies, les devis et mémoires, et des fiches notant les interventions les unes à la suite des autres, qui deviendront, après la Seconde Guerre mondiale, les « fiches de santé » des peintures du Louvre, sur le modèles des dossiers médicaux. 


\section{Conclusion}

La fin du XVIII ${ }^{\mathrm{e}}$ siècle, avec l'émergence de la profession de restaurateur de tableaux, la création des musées et l'ouverture des collections au grand public, est la période où se constitue un début de documentation de la restauration des peintures (constats détaillés, devis et mémoires, procès-verbaux de l'administration). Ce type de documentation où dominent les éléments comptables perdure au XIX ${ }^{\mathrm{e}}$ siècle, et on a conservé peu de documents visuels permettant de juger des interventions anciennes. L'exemple rare de la restauration du retable du Jugement dernier au Louvre montre cependant le désir de disposer d'images documentaires grâce au développement de la photographie, mais le coût encore important de cette technique freine sans doute son usage. C'est donc surtout au cours du $\mathrm{XX}^{\mathrm{e}}$ siècle que vont se généraliser de nouvelles pratiques documentaires (constitution de dossiers, suivi régulier des œuvres) et un recours aux techniques modernes de la photographie et d'autres types d'imagerie scientifique (rayons X, ultra-violets).
Notes

1. Dès 1775, Joseph Ferdinand Godefroid, peintre et restaurateur, expose cette méthode de travail au comte d'Angiviller. Puis, au moment de la création du Musée central des arts au Louvre en 1793, l'idée est reprise par les principaux acteurs de la restauration, qu'il s'agisse de restaurateurs comme Jean-Michel Picault, François-Toussaint Hacquin ou l'expert et marchand Jean-Baptiste Pierre Le Brun. L'obligation du constat fait enfin l'objet d'un article propre (article 7) dans le nouveau règlement du Louvre (alors Musée central des arts) en 1797 : « Nul tableau, nul objet d'art, ne sera mis en restauration qu'en vertu d'une délibération du conseil qui constatera l'état de l'objet à restaurer ; l'état de l'objet sera constaté également après la restauration et consigné dans le registre des délibérations ( ) ", Organisation de l'administration du Musée central des arts, AN, F171059, dos 23 ; lu lors de la séance du 27 janvier 1797 ( 8 pluviose an V). Voir Cantarel-Besson, 1992.

2. Voir par exemple les informations assez détaillées données par le restaurateur Godefroid en 1785-1786 à propos de tableaux de Lesueur ; pour Sainte Scholastique (inv. 8022), « a levé quantité de repeints lourds et mal faits et chargés qui couvraient en grande partie les figures ; il a découvert d'autres objets cachés par les repeints et refait en partie les allonges de haut et de bas et repointillé beaucoup d'endroits dans les figures» (AN, O1 $1922 \mathrm{~A}$, doc. 6).

3. Voir Coural et Gerin-Pierre, 2008.

4. Voir par exemple Étienne, 2011.

5. Rapport sur la restauration du tableau de Raphaël connu sous le nom de la Vierge de Foligno, adopté par les classes des sciences mathématiques et physiques, et de littérature et beaux-arts, dans les séances des 1 et 3 pluviose an X par les citoyens Guyton, Vincent, Taunay et Berthollet, Paris, Baudoin, imprimeur de l'Institut national, pluviose an X (1802). Rendu nécessaire par le contexte particulier de ces restaurations sur des œuvres étrangères « saisies » par les armées françaises, et qu'il fallait bien justifier, ce rapport ne pouvait faire école car il détaillait des pratiques (particulièrement pour la transposition) dont les restaurateurs voulaient conserver au moins partiellement le « secret professionnel ». Et il fut réalisé sans le consentement du restaurateur de support, F. T. Hacquin. Voir Étienne, 2012.

6. Voir Cantarel-Besson, 1981 et 1992.

7. Voir Gerin-Pierre, 2008.

8. Voir Chaizemartin et Cabillic, 2008.

9. Très souvent, seul le terme

" restauré » est employé, sans autres précisions, pour la couche picturale ; et pour les supports, les opérations de refixage, de rentoilage ou d'" enlevage » (transposition) sont simplement mentionnées, sans plus de détails.

10. On peut citer parmi les plus importants ceux de Burtin (1808), Edwards (1819), Bedotti (1837), Köster (1827), Horsin-Déon (1851).

11. Ce plafond avait été enlevé du Palais des Doges et emporté par les troupes françaises en 1797, puis employé pour décorer un plafond du château de Versailles en 1814 : les dimensions étant trop petites, il avait été agrandi et on avait recoupé les groupes de figures ; endommagé par un gros orage en 1858, il avait été emmené au Louvre pour être restauré, et on en avait profité pour le remettre dans son état original.

12. AN, fonds des AMN, P16, 19 mars 1861 .

13. Victime dès 1851 de nombreuses attaques dans la presse pour ses restaurations de tableaux célèbres (dont Les Noces de Cana de Véronèse et les Rubens de la galerie du Luxembourg), Villot dut finalement démissionner de ses fonctions en 1861 .

14. AN, fonds des AMN, P16, 12 avril 1860.

15. Pour l'histoire détaillée de cette restauration, voir Gerin-Pierre, 2010, p. $86-95$.

16. Ibid.

17. AN, fonds des AMN, P16, 1875-1878.

18. Archives des Hospices civils de Beaune, VIII P1, recueil intitulé « Le tableau Le Jugement dernier/Recueil de correspondances, délibérations, notes provenant de l'administration des Hospices documents divers donnés par plusieurs personnes, constituant l'historique de la restauration de cet objet d'art de R. van der Weyden/Recueil divisé en 5 parties ». 19. Sans numéro d'inventaire ou de classement actuellement.

20. MAP, Dossier Hôtel-Dieu/Hospices de Beaune, $\mathrm{n}^{\circ}$ 21/072.
21. Photographies réalisées en 1882 , si l'on en croit la facture figurant dans le recueil consacré à la restauration, car il semblerait que Reiset, en raison de ses problèmes de santé, puis de l'organisation de l'exposition du retable au Louvre pendant l'exposition universelle de 1878 , n'ait pas eu le temps d'organiser une séance de prises de vue, et que la commission des Hospices n'ait finalement commandé ces clichés qu'en 1882, au moment de l'ouverture au public du petit musée consacré à l'exposition du retable après sa restauration.

22. Le 6 avril 1875, " la commission décide que l'un des panneaux du jugement dernier sera démonté et photographié ensuite par M. Cochey en présence de M. de Vergnette-Lamotte ", Archives des Hospices civils de Beaune, Registre des délibérations, II P1.

23. "J'ai fait photographier hier par M. Braun les deux faces de la peinture. L'opération a, je crois, bien réussi. Mais je n'ai pas les épreuves. Je vous en enverrai une ou deux et je pense que vous les verrez avec plaisir », Lettre de Reiset à Cyrod, 1 6 juin 1876 (AMN P16, 1875-1878 et Archives des Hospices civils de Beaune, VIII P1, 1869-1914).

24. Lettre du 11 janvier 1877 , ibid.

25. 18 janvier 1877 , ibid.

26. Lettre de Reiset à Cyrod, 3 avril 1877, ibid.

27. Il s'agit des dessins préparatoires sous-jacents que l'on peut aujourd'hui voir grâce à la technique de la réflectographie infra-rouge.

28. Archives des Hospices civils de Beaune, VIII P1, 1869-1814.

29. Lettre de Reiset à Cyrod, 10 mai 1876 , ibid.

30. Lettre de Briotet à Cyrod, 8 juillet 1878, Archives des Hospices civils de Beaune, VIII P1.

31. Il est frappant en effet de voir cette question revenir plusieurs fois dans la correspondance entre Reiset et Cyrod : les deux premières épreuves réalisées par la maison Braun ont été gratuites apparemment («je tâcherai que cela vous coûte aussi peu que possible. Les épreuves que j'ai fait faire et que je vous ai envoyées n'ont rien coûté. C'est la maison Braun qui les a faites et qui n'a rien voulu recevoir de moi », lettre de Reiset à Cyrod du 19 janvier 
1877). Mais pour les photographies du reste du retable avant intervention, Reiset envoie à Cyrod le 3 avril 1877 une facture du photographe Berthier de $260 \mathrm{~F}$ (relativement importante pour l'époque), en précisant que le travail est bien fait et que c'est pour lui le prix le plus bas possible; et la note du photographe de Beaune, en 1882, pour les épreuves après restauration, est de $570 \mathrm{~F}$ (2 séries de grandes plaques et 2 séries de petites). Enfin, interrogé par Cyrod sur le meilleur procédé à choisir pour faire réaliser un facsimilé du retable restauré, Reiset répond qu'il ne connait pas bien toutes les nouvelles techniques de reproduction, mais qu'il préfèrerait la photographie, si ce n'était si coûteux : "Je préfèrerais de beaucoup quant à moi de bonnes épreuves photographiques, 2008.

\section{Bibliographie sélective}

Cabillic I., 2008, «Jean-Gabriel Goulinat, chef de l'atelier de restauration des peintures des musées nationaux (1935 à 1971) ", Techné, n 27-28, p. 92-98.

Cantarel-Besson Y., 1981, La naissance du musée du Louvre : la politique muséologique sous la Révolution d'après les archives des musées nationaux, Paris, éditions RMN.

Cantarel-Besson Y., 1992, Musée du Louvre (janvier 1797-juin 1798) procès-verbaux du Conseil d'administration du Musée Central des Arts, Paris, éditions RMN.

Chaizemartin J. et Cabillic I., 2008, «Les commissions de restauration des peintures des Musées nationaux de 1882 à 1937 », Techné, n 27-28, p. 156-159.

Coural N. et Gerin-Pierre C., 2008, «La restauration du carton de l'École d'Athènes de Raphael en 1797 et la politique de conservation de l'administration du Louvre ", Techné, n 27-28, p 113-118.

Emile-Mâle G., 1957, «Jean-Baptiste Pierre Le Brun (1748-1813), son rôle dans l'histoire de la restauration des tableaux du Louvre ", dans Paris et Île de France, Mémoires, T. VIII, Paris, p. 153-171.

Emile-Mâle G., 2008, Pour une histoire de la restauration des peintures en France, études réunies par Bergeon-Langle $\mathrm{S}$., coordination scientifique Toscano G., Paris, Somogy, INP.

Étienne N., 2011, «Polémique et politique. La restauration dans l'espace public à Paris autour de 1800 », Histoire de l'art, p. 9-17.

Étienne N., 2012, La restauration des peintures à Paris (1750-1815), pratiques et discours sur la matérialité des æeuvres d'art, Presses Universitaires de Rennes, Rennes.

Gerin-Pierre C., 2008, « Les commissairesexperts au musée du Louvre, de la Révolution à la monarchie de juillet ", Techné, n 27-28, p. 60-70.

Gerin-Pierre C., 2011, « La première restauration du retable de Beaune au Louvre (1875-1878) ", Techné, $\mathrm{n}^{\circ} 33$, p. $86-95$.

Goulinat A.-M., 1974, Jean-Gabriel Goulinat, sa vie, sa carrière, Carrefour des lettres, Paris.

Philippe E., 2008, « Innover, connaître et transmettre, l'art de la restauration selon François-Toussaint Hacquin (1756-1832) », Techné, n 27-28, p. 53-59.

Savoy B., 1999, « Et comment tout cela sera-t-il conservé à Paris ? Les réactions allemandes aux saisies d'œuvres d'art et de science opérées par la France autour de 1800 ", Écrire l'histoire de l'art. FranceAllemagne 1750-1920, Revue germanique internationale, 13, p. 107-130.
Savoy B., 2003, Patrimoine annexé. Les bien culturels saisis par la France en Allemagne autour de 1800, Paris, Maison des Sciences de l'homme, collection Passages, 2 vol.

Volle N., 2008, « Titien et ses restaurateurs, Jupiter et Antiope, un tableau palimpseste », Techné, n 27-28, p. 21-28.

Veronee-Verhaegen, N., 1973, L'Hôtel-Dieu de Beaune (Les primitifs flamands, corpus de la peinture des Anciens Pays-Bas méridionaux au $X V^{e}$ siècle, 13), Bruxelles.

\section{Sources}

Archives nationales $(A N)$ :

Série O1 (maison du Roi).

Série F17 (travaux dépendant du ministère de l'Instruction publique sous la Révolution) et F21 (comptabilité Révolution et Empire).

Archives nationales, fonds des Archives des musées nationaux $(A M N)$ :

Série P16 (restauration des peintures).

Série 1BB 4-6 (procès- verbaux de l'administration du Louvre sous le Directoire et le Consulat).

Médiathèque de l'Architecture et du Patrimoine $(M A P)$ :

Dossier Hôtel-Dieu/Hospice de Beaune, $\mathrm{n}^{\circ}$ 21/072).

Archives des Hospices civils de Beaune:

Série II P1 et II P2 (registres des délibérations).

Série VIII P1 (correspondance, 1869-1914). 